\title{
A new method of administering local anesthesia for calf disbudding: Findings from a comparative on-farm study in New Zealand
}

\author{
A. J. Bates, ${ }^{1,2 *}$ M. A. Sutherland ${ }^{3}$ F. Chapple, ${ }^{2}$ S. K. Dowling, ${ }^{3}$ A. P. Johnson, ${ }^{2}$ B. Saldias, ${ }^{1}$ and J. Singh ${ }^{2}$ \\ ${ }^{1}$ Vetlife, Centre for Dairy Excellence, Geraldine 7930, New Zealand \\ ${ }^{2}$ Vetlife Temuka, Temuka 7920, New Zealand \\ ${ }^{3} \mathrm{AgResearch,} \mathrm{Ruakura} \mathrm{Research} \mathrm{Centre,} \mathrm{Hamilton} \mathrm{3240,} \mathrm{New} \mathrm{Zealand}$
}

\section{ABSTRACT}

Anesthesia of the horn bud for calf disbudding commonly is attained by injection of local anesthetic over branches of the cornual nerve, with anesthesia achieved in 3 to $20 \mathrm{~min}$. With experienced and trained staff, this method is effective in 88 to $100 \%$ of calves. Variability in response and time of onset can compromise calf welfare if calves are disbudded before anesthesia is attained. Proposed legislative reliance on effective local anesthetic as the minimal method of pain relief for calves at disbudding means that administration of local anesthetic must achieve a repeatable level and rapid onset of analgesia. We describe an alternative method of local anesthesia administration that uses local site infiltration of anesthetic over the horn bud. However, this method has not yet been scientifically validated. This study assessed differences between disbudding using the cornual nerve block and disbudding with local anesthesia administered by local site infiltration. Efficacy of local anesthesia was assessed at 30-s intervals after administration by absence of reaction to 3 consecutive needle pricks over the horn buds. Behavior indicating pain was assessed during disbudding and scored from 0 to 3 . Calf behavior was also recorded for $3 \mathrm{~h}$ after disbudding. Accelerometer data loggers were fitted to each calf for $24 \mathrm{~h}$ before and after disbudding to assess lying and standing times. Median time to cutaneous desensitization for local infiltration was $60 \mathrm{~s}$ compared with $225 \mathrm{~s}$ for cornual nerve block, and the variance in time to desensitization was less with local infiltration. Calves disbudded under cornual block had a larger behavioral response (indicated by a graded aversive body reaction) than calves disbudded under local infiltration. A multivariable model predicted that the mean body reaction score would be 0.6 for calves disbudded under local infiltration and 1.2 for calves

Received May 9, 2018.

Accepted November 14, 2018.

*Corresponding author: andrew.bates@vetlife.co.nz disbudded under cornual block. There was no difference in any behaviors between the treatment groups in the $3 \mathrm{~h}$ after disbudding. Method of analgesia had no effect on lying time over the $24 \mathrm{~h}$ after disbudding. In this study, local infiltration was at least as effective in providing analgesia for disbudding as the cornual nerve block. Our results suggest that a more consistent, effective level of analgesia during disbudding was achieved using local infiltration and that there was no difference in postoperative expressions of pain.

Key words: disbudding, calf, cornual, local, infiltration

\section{INTRODUCTION}

The major dairy breeds are naturally horned, and in mature cattle this presents a risk to animals and people (Stock et al., 2013). Disbudding refers to the destruction of free-floating immature horn tissue (horn "buds" growing from the skin), from which the horns of the animal subsequently develop (Mellor and Stafford, 2004). There is evidence that disbudding is less painful than amputation dehorning (Petrie et al., 1996; Stilwell et al., 2007). Within the major dairy-producing countries, disbudding is usually performed using a hot iron when calves are 3 to 6 wk of age and horn buds are 5 to $10 \mathrm{~mm}$ long (Bates et al., 2016; Winder et al., 2017). Caustic paste is used in around $20 \%$ of disbudded calves in the European Union (Gottardo et al., 2015) and 16\% of those in the United States (Adams et al., 2015).

There is abundant evidence in the scientific literature of the benefits of providing local anesthesia and systemic analgesia at the time of disbudding (Stafford and Mellor, 2011; Stock et al., 2013; Bates et al., 2015) and an increasing social understanding of the pain associated with disbudding (Green and Mellor, 2011; Robbins et al., 2015; Ventura et al., 2015). This has led to a legislative and social environment where the provision of analgesia at disbudding is obligatory (Croney and Anthony, 2010; Lundmark et al., 2014; Winder et al., 2017), including within New Zealand (NZ), where the use of local anesthetic at disbudding is mandatory beginning in 2019. The cornual nerve block is currently 
the accepted method of administering local anesthesia for disbudding in calves (Grøndahl-Nielsen et al., 1999; Fierheller et al., 2012), and postoperative analgesia is provided by nonsteroidal anti-inflammatory drugs (Stafford and Mellor, 2011). Ring blocks and cornual blocks with local anesthetic placed medial to the horn bud have also been described (Stock et al., 2013).

Within the United States, Canada, NZ, and much of the European Union, it is common practice for disbudding to be performed on standing calves by non-veterinarians (Gottardo et al., 2015; Bates et al., 2016; Winder et al., 2018). Mandatory use of local anesthetic at disbudding will be effective in improving calf welfare only if an effective nerve block is achieved. Correct application of a cornual nerve block requires technical training on the injection site and technique (Winder et al., 2018). The volume and the amount of time needed between injection and the procedure also needs to be established during training, with Stock et al. (2013) quoting a median of $5 \mathrm{~mL}$ per bud (range $=3-5 \mathrm{~mL}$ ) and a median time of $10 \mathrm{~min}$ (range $=$ 5-20 min). Winder et al. (2018) demonstrated that effective training in administering a cornual block in standing calves could be attained using either online or hands-on training techniques. Based on the elimination of behavioral responses to disbudding during the first $5 \mathrm{~s}$ of the procedure, an effective corneal nerve block was successfully achieved by 75 and $91 \%$ of the hands-on and online trained participants, respectively. In this study, a 10-min delay was used between anesthetic block administration and disbudding. In many seasonal-calving herds, large numbers of calves require disbudding over a short time period, and in NZ there is often a requirement to disbud groups of 200 to 400 calves during a single visit. In this situation, a delay between block administration and procedure leads to double handling of calves or requires multiple restraining crates to avoid downtime. This is often impractical, and the extra handling can cause additional stress to the animal.

The present paper describes an alternative and, to our knowledge, previously undescribed method of administering a local anesthetic block to the horn bud (local infiltration block) that requires a much shorter delay and less technical skill in administration. For horn buds that are not yet attached to the skull, the method consists of injecting local anesthetic $(\sim 1 \mathrm{~mL})$ directly over the horn bud, which results in the local anesthetic diffusing in a bubble or bleb around the caudomedial or rostromedial aspect of the horn bud. For larger buds that have attached to the underlying bone, local anesthetic is injected laterally $(1 \mathrm{~mL})$ and caudally $(1 \mathrm{~mL})$ as close to the horn bud as possible. Similar to the methodology used for the unattached horn buds, the local anesthetic diffuses in a bubble or bleb around the caudomedial or rostromedial aspect of the horn bud. Unlike the multiple injections required for a ring block (Fierheller et al., 2012), this method involves 1 or at most 2 injection sites per horn bud. Our null hypothesis was that there would be no difference in the behavioral signs of pain and discomfort during disbudding and in the $3 \mathrm{~h}$ afterward and no difference in the lying and standing times in the $24 \mathrm{~h}$ after disbudding between calves disbudded under a cornual block and calves disbudded under the local infiltration block.

\section{MATERIALS AND METHODS}

\section{Animal Use}

This trial was conducted between August and September 2017 on 2 commercial seasonal-calving pastoral dairy farms (A and B) in Canterbury, South Island, NZ. Use of animals and all procedures was preapproved by the Ruakura (NZ) Animal Ethics Committee (application no. 14287).

\section{Housing and Management}

All calves were born at pasture and brought into the rearing facility within $24 \mathrm{~h}$ of birth, and their navels were sprayed with iodine tincture spray containing 2.5\% iodine (Ethical Agents, Manukau, NZ). On farm A, calves were housed in groups of approximately 40 calves/pen in purpose-built sheds with solid walls up to $1.5 \mathrm{~m}$ high. On farm B, calves were housed in groups of up to 10 in covered, open-front bay sheds with solid walls up to $1.5 \mathrm{~m}$ high. All calves were bedded on wood chips that were cleaned daily and topped up weekly. Male and female Jersey-Friesian crossbred calves were used, and all calves remained on the farm of origin for the period of the study.

\section{Nutrition}

For the first $24 \mathrm{~h}$ after birth, all calves were fed pooled colostrum in 2 feeds of 1.5 to $2.0 \mathrm{~L} /$ calf per feed, harvested from cows calving that day. For the next $3 \mathrm{~d}$, calves were fed $2 \mathrm{~L}$ of pooled colostrum twice daily from cows between 1 and $4 \mathrm{~d}$ calved. Thereafter, calves were fed pooled colostrum and nonsaleable milk and had access to perennial ryegrass (Lolium perenne) hay, water, and calf meal (Calf-Pro20\%, Winslow Feeds, Tinwald, Ashburton, NZ; and NRM GrowUp 20\%, NRM, Canterbury, NZ). This contained $20 \% \mathrm{CP}$ and $13 \mathrm{MJ}$ of $\mathrm{ME} / \mathrm{kg}$ of DM. On farm A, calves were fed individually via an automated milk feeding system (rEID Calf Feeder, A\&D Reid, Temuka, NZ), which allocated up 
Table 1. Description of the treatment groups used in analysis of the efficacy of local anesthetic infiltration and cornual nerve block on the pain arising from calf disbudding

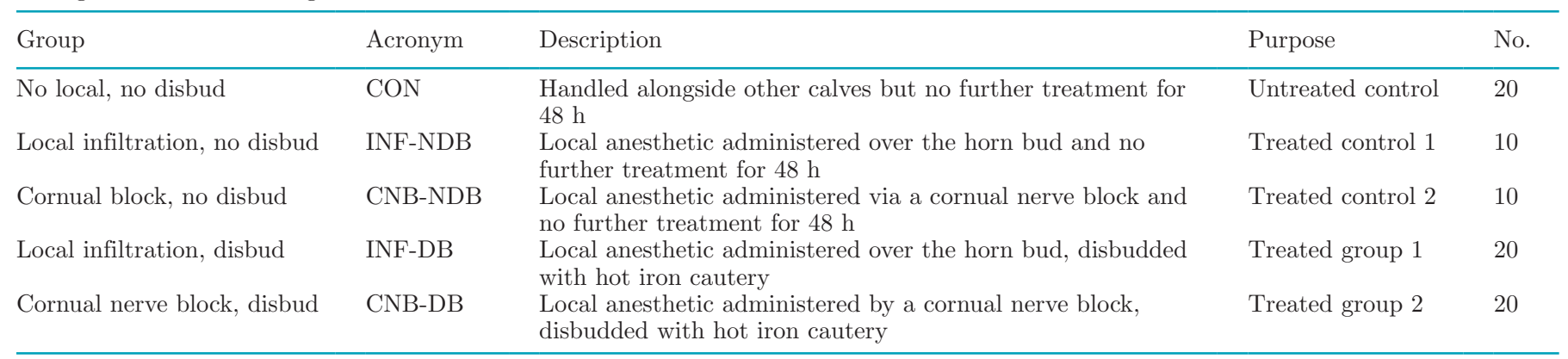

to 4 feeds/d of up to $1.5 \mathrm{~L}$ of milk/feed. On farm B, calves were fed 2 equal feeds $(0700$ and $1500 \mathrm{~h})$ increasing from $2 \mathrm{~L} /$ feed over the first $21 \mathrm{~d}$ and remaining at 6 to $8 \mathrm{~L} / \mathrm{d}$ for the duration of the study.

\section{Enrollment}

The 2 study farms were chosen on a convenience basis from farms serviced by Vetlife Ltd. (Temuka, New Zealand). Calves with no visible signs of illness were scheduled for routine disbudding from 4 wk of age at biweekly farm visits where 40 calves were disbudded per visit. For each farm a scheduled visit (farm A: Aug. 29, 2017; farm B: Sep. 5, 2017) was chosen using the random number generator in Microsoft Excel (Microsoft Corp., Redmond, WA) to serve as the study group for that farm. The mean age was $31 \mathrm{~d}(95 \% \mathrm{CI}=28-34)$, the mean proportion of Friesian genetics was 0.60 (95\% $\mathrm{CI}=0.58-0.61)$, and $16 \%(95 \% \mathrm{CI}=10.5-22.0)$ of study calves were uncastrated males.

\section{Treatment Groups}

Twenty-four hours before disbudding, all study calves had Hobo Pendant G accelerometers (64k, Onset Computer Corp., Bourne, MA) attached to the rear right leg and were marked with a unique study number spraypainted using nonirritant stock marker (Tell-Tail; FIL, Mt. Maunganui South, NZ) on the back and left and right flanks. Accelerometers were set at 1-min intervals recording the $\mathrm{y}$ - and $\mathrm{z}$-axes, placed in a durable fabric pouch, and strapped onto the lateral side of the hind leg above the metatarsophalangeal joint, as described in Sutherland et al. (2017).

No calves were treated or disbudded at this visit, but calves were allocated using restricted randomization and a random number generator from Microsoft Excel (Microsoft Corp.) to a treatment group for the following day, as detailed in Table 1. Each calf was fitted with a colored neckband to identify treatment group (Shoof NZ Ltd., Cambridge, NZ), but all farm and veterinary staff were blinded to the color coding of the treatment groups, which was known only to the lead investigator. All calves were managed identically during the study period.

On the morning of disbudding, calves were deprived of milk but had access to meal and roughage. Each pen of calves was divided into a front and back area using mobile gates, and calves were moved into the back area. A mobile calf crate was set up at the front of the rear calf pen, and calves were individually moved into the crate and restrained. All local anesthetic treatments were administered by the same trained veterinarian (the lead author).

The study design was a $2 \times 2+1$ factorial with disbudding and method of local anesthesia as the factors and with the following groups. Control $(\mathbf{C O N})$ calves $(\mathrm{n}=20)$ were restrained in the calf crate for 30 to $60 \mathrm{~s}$, and their horn buds were touched with a single digit at $30 \mathrm{~s}$ intervals but not disbudded. For the calves in the local infiltration groups (local anesthetic administered over the horn bud and no further treatment for $48 \mathrm{~h}$, INF-NDB, $\mathrm{n}=10$; local anesthetic administered over the horn bud and disbudding with hot iron cautery, INF-DB, $\mathrm{n}=20$ ), analgesia was administered to each calf by subcutaneous infiltration of $40 \mathrm{mg}$ of lidocaine hydrochloride $(2 \mathrm{~mL}$ of Nopaine $2 \%$; Phoenix Pharm Distributors, Auckland, NZ) per horn bud (Figure 1). To standardize the approach in the present study, local anesthetic was injected laterally $(1 \mathrm{~mL})$ and caudally $(1 \mathrm{~mL})$ as close to each horn bud as possible using an 18-gauge 2.5-cm needle (Rurtec, Hamilton, NZ). Once the local anesthetic was injected, it diffused in a bubble or bleb around the caudomedial or rostromedial aspect of the horn bud in a crescent-shaped pattern.

For the calves in the cornual nerve block group (cornual nerve block with no further treatment for 48 h, CNB-NDB, $\mathrm{n}=10$; cornual nerve block and disbudding with hot iron cautery, CNB-DB, $\mathrm{n}=20$ ), analgesia was administered by injecting $5 \mathrm{~mL}$ of $2 \%$ lidocaine hydrochloride (Nopaine 2\%; Phoenix Pharm Distributors) per horn bud subcutaneously using an 
18-gauge 2.5-cm needle (Rurtec) around the cornual nerve (located along the occipital groove midway between the horn bud and the eye) as a cornual nerve block. Four milliliters was injected at this location after ensuring that the needle was not in a blood vessel, and the remainder was infiltrated through the tissues on removal and repositioning of the needle subcutaneously over the frontal crest (Skarda, 1996; Fierheller et al., 2012).

Behavioral observations during disbudding were recorded by the same veterinary technician for all calves. Observations of calf behavior during the $3 \mathrm{~h}$ after disbudding were recorded by the same team of 4 veterinarians trained in behavioral observation. All observers were blinded to the treatment group of the calves.

Control calves received no further treatment but were restrained in the calf crate for 30 to $60 \mathrm{~s}$ and their horn buds were touched with a single digit at 30-s intervals but not disbudded. In all groups that received local anesthetic (INF-NDB, CNB-NDB, INF-DB, CNB-DB), efficacy of the local anesthesia was assessed at 30-s intervals after administration and judged by reaction to a fresh 1.5-inch, 22-gauge sterile needle pricked over the horn buds. The horn bud was approached caudally and dorsally to reduce reaction from the approach of the handler. Initially, the horn buds were digitally massaged and if no reaction was evident (head movement away from the stimulus), a single needle prick was made over the horn bud. If no reaction was evident, second and third needle pricks were made in a similar manner. Reaction at any point triggered another stand-down period of $30 \mathrm{~s}$ with a calf having to show no reaction to a total of 3 consecutive needle pricks to be submitted for disbudding.

A

B

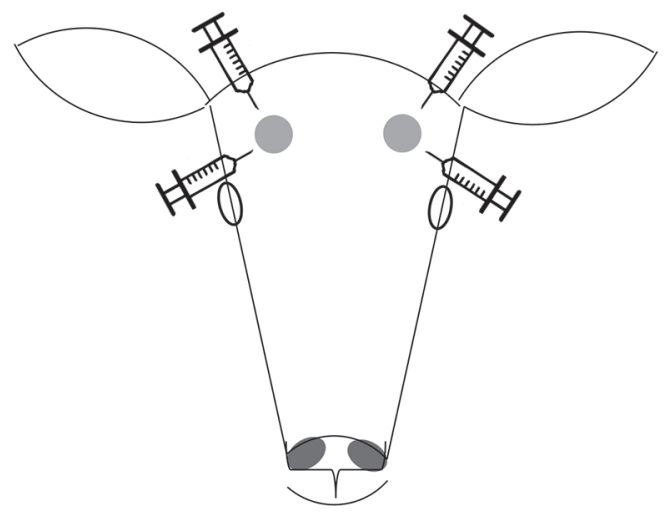

On attainment of anesthesia, the horn buds of calves in groups INF-NDB and CNB-NDB were massaged manually for $12 \mathrm{~s}$ with the thumb and index finger but calves were not disbudded, whereas calves in the groups INF-DB and CNB-DB were disbudded. For INF-DB and CNB-DB calves, on attainment of anesthesia, disbudding was performed using a cordless gas-fueled hot cautery calf dehorner (Portasol Mark 3 debudder; Portasol, Elmira, OR) and the wound was sprayed with a topical aerosol of oxytetracycline (Aerotet-Forte; Birbac NZ, Auckland, NZ). Contact time between the hot iron and the bud was standardized to 12 to $15 \mathrm{~s}$ per bud, and the center was scooped out at the end with the disbudding iron. On completion of treatment, the colored neckband was removed and each calf was released from the calf crate into the front half of the pen. At the completion of disbudding, the temporary gates dividing the pen into front and back halves were removed. Thereafter, all calves were treated identically per normal farm management practices.

\section{Outcomes}

Time to Skin Desensitization. For CON calves, the time from securing the calf within the crate to absence of reaction to digital contact with the horn bud was recorded. For the remaining groups, the time from administration of local anesthetic to skin desensitization was recorded for each horn bud.

Behavior During Disbudding. Body response was classified on a scale of 0 to 3 using the definitions given in Table 2, modified from Stewart et al. (2008). The frequency of vocalizations and defecations was also recorded for each calf. A second observer was present

Figure 1. Diagram of administration of local anesthetic into the horn bud region by the local infiltration technique used in the present study. (A) Syringes indicate the rostromedial and caudomedial locations around the horn bud where the needle was inserted. (B) Dotted lines indicate how the local anesthetic disperses in a crescent pattern, laterally and caudally, when it is injected. 
Table 2. Behaviors recorded during and after disbudding and massaging

\begin{tabular}{ll}
\hline Behavior & Description of body response \\
\hline $\begin{array}{l}\text { During disbudding } \\
\text { Body reaction score }\end{array}$ & \\
0 & No response, slight movement of body, tail wagging \\
1 & Mild struggling, no foot stamping \\
2 & Struggling with hind and front limbs \\
3 & Massive struggling involving whole body \\
After disbudding & Vigorous shaking of the head around a rostral to caudal axis \\
Head shaking & Scratching the top of the head with a hind foot; includes attempts where hind foot does not make contact \\
Head scratching & with the head \\
Head rubbing & Rubbing the top of the head against the side of the pen or other calf with an up-down or side-to-side \\
melf-grooming & Calf's tongue is out of its mouth and in contact with its own body \\
Running (locomotor play) & Includes trotting (2-beat gait), cantering (3-beat gait), and galloping (fast 4-beat gait) with forward or \\
& sideways movement \\
\hline
\end{tabular}

at both farms to determine the level of interobserver agreement.

Behavior After Disbudding. After disbudding, 2 trained observers blinded to the treatment status of the calves recorded calf behavior for all calves using the definitions in Table 2 (Grøndahl-Nielsen et al., 1999; Faulkner and Weary 2000). Observations were recorded against the calves' spray-painted and ear tag numbers. Each calf was observed for a period of $45 \mathrm{~s}$ immediately after exit from the calf crate and then every $20 \mathrm{~min}$ until $3 \mathrm{~h}$ after disbudding had elapsed. At each observation period, the frequency of the observed behaviors in Table 2 was recorded. These behaviors were chosen because head shaking, scratching, and rubbing have been shown to indicate postdisbudding pain in previous studies (reviewed by Stafford and Mellor, 2005).

Lying and Standing Behavior After Disbudding. Data loggers were removed $24 \mathrm{~h}$ after disbudding. Accelerometers were initialized and downloaded using HOBOware Pro software (version 3.7.2; Onset Computer Corp., Bourne, MA). G-force readings were converted into binary values (e.g., lying $=0$, standing $=1$ ), and daily $(\mathrm{min} / \mathrm{d})$ summaries of lying time and lying-bout frequency (number of bouts/d) were calculated in SAS 9.3 (SAS Institute Inc., Cary, NC) using a code designed for this purpose (UBC AWP, 2015) and validated by Bonk et al. (2013) for use in calves.

\section{Statistical Analysis}

Power Analysis. A prestudy power analysis was conducted using data from Graf and Senn (1999) on the frequency postdisbudding of head shakes and from Heinrich et al. (2010) on ear flicks. A modified version of Lehr's equation (Lehr, 1992) was used to account for the Poisson distribution of count data (van Belle, 2008). It was estimated that if the difference between control and treatment in counts of outcome was at least
$50 \%$, groups of 20 calves would maintain power above 0.84 at a $95 \%$ confidence interval for counts ranging from 4 to 10 in the control group, whereas groups of 10 would be sufficient to distinguish counts ranging from 6 to 10 in the control group. All data were recorded on purpose-designed paper records and then entered manually into Microsoft Excel (Microsoft Corp.) and then exported into $\mathrm{R}$ version 3.3.2 (R Foundation for Statistical Computing, Vienna, Austria) for statistical analysis.

Independent Variables. Treatment group was forced into all models with calf as the experimental unit. To increase power and aid interpretation, treatment groups were aggregated together where appropriate. For example, to determine the overall success of anesthesia for disbudding, where there was no significant difference between the control groups (CON, INFNDB, and CNB-NDB), these were aggregated together to form a 3-component categorical variable: calves not disbudded $[\mathrm{n}=40$; some receiving no local anesthetic $(\mathrm{n}=20)$, some receiving local infiltration of anesthetic $(\mathrm{n}=10)$, and some receiving a cornual block $(\mathrm{n}=10)]$, calves disbudded under local infiltration of anesthetic $(\mathrm{n}=20)$, and calves disbudded under cornual block ( $\mathrm{n}$ $=20)$.

Farm was recorded as a categorical variable, calf sex as a categorical variable, age in days as a continuous variable, and breed as the proportion of Friesian genetics (continuous). For each multivariable model, all independent variables were assessed for correlation. Pearson correlation coefficient was calculated for normally distributed continuous variables, Spearman's coefficient for nonnormally distributed continuous variables, and Goodman and Kruskal's tau for categorical variables. Where a correlation of $>0.2$ was found, a variance inflation factor to assess collinearity was calculated using auxiliary regressions of one of the correlated variables on the remaining explanatory variables in the model. If 
the variance inflation factor was $>10$ or if the value of the remaining coefficients changed by more than $10 \%$ when rerunning the model without the variable, the collinear variables were assessed for biological plausibility and the least useful were discarded from the final model. Variables that were significant at the univariable level at $P<0.20$ were entered by hand within each multivariable model and retained if identified as confounders, if a Wald test for the variable gave a $P$ value $<0.05$, or if a likelihood test for inclusion of the variable category was significant $(P<0.05)$.

Dependent Variables: Time to Skin Desensitization. Time for cutaneous desensitization following administration of local anesthetic was reported for each calf (average for both horn buds) as a discrete variable, reported from 0 to 300 in 30 -s intervals. Univariable analysis for the effect of treatment group on time to skin desensitization was conducted using a KruskalWallis rank-sum test followed by a pairwise Wilcoxon rank-sum test with Holm-Bonferroni adjustment for multiple comparisons to allow relative comparison of the effect of individual treatments. Data visualization, Bartlett, Levene, and Fligner-Killeen tests for homogeneity of variance all indicated heterogeneity of variance between treatment groups $(P<0.001)$. As time was measured in discrete intervals of $30 \mathrm{~s}$, the probability of a successful block was estimated for each discrete time interval using a multivariable binomial general linear model. A logit link function was used because it resulted in a lower residual deviance than a complementary log-log link function. Complete separation of the data meant that it was necessary to use penalized maximum likelihood estimation to estimate parameters (Heinze and Schemper, 2002).

Dependent Variables: Behavior During Disbudding. Within each calf, body response to disbudding for each horn bud differed by no more than 1 unit; therefore, calf body response during disbudding was averaged for both horn buds for that calf and reported as an ordinal variable from 0 to 3 in intervals of 0.5 . Median average body response score for each calf was compared between groups using a Kruskal-Wallis ranksum test followed by a pairwise Wilcoxon rank-sum test with Holm-Bonferroni adjustment for multiple comparisons to allow relative comparison of the effect of individual treatments.

All control groups (CON, INF-NDB, CNB-NDB) recorded average body response up to and including 1.0; therefore, calves were also categorized as having a body response of $\leq 1.0$ (considered successful anesthesia) or greater than 1.0 (considered unsuccessful anesthesia). Univariable analysis for the effect of treatment on the proportion of calves with successful anesthesia (body response score $\leq 1.0$ ) was conducted using Fisher's exact test followed by a pairwise Fisher test with Holm-Bonferroni adjustment for multiple comparisons to allow relative comparison of the effect of individual treatments.

Multivariable ordinal logistic models were used to explore the effect of the combined independent variables on the average calf body response score during disbudding. Models with a logistic, probit, and cumulative logistic link function were constructed, and the model with the lowest Akaike fixed and information criteria (AIC) was chosen. The assumption that the coefficient values were appropriate across all levels of the ordinal response (parallel regression assumption) was assessed graphically (Harrell, 2001).

Spearman's rank correlation coefficient was determined for the behavioral score during disbudding recorded by each observer. Values of $>0.9$ were taken to indicate acceptable agreement between observers.

Dependent Variables: Behavior in the $3 \mathrm{~h}$ After Disbudding. Behavior after disbudding was reported as the frequency of observed counts for each behavior over the entire period of observation and at each observation time. For regression analysis, counts were standardized to a denominator of 20 calves to account for the different number of calves in groups INFNDB and CNB-NDB from the groups CON, INF-DB, and CNB-DB. For all the dependent variables, variance greater than the mean indicated possible overdispersion. Consequently, for each dependent variable, Poisson and negative binomial multivariable models were compared using a likelihood ratio test.

For the analysis of the total count of each behavior, all variables in each model were treated as fixed. For the analysis of the count of observations at each observation time, calf was included as a random intercept within the model to account for repeated observations on the same calf. The proportion of the total variance for repeat measures on the same calf was calculated as the intraclass correlation coefficient. The effect of observation time was nonlinear, and models where observation time was forced into the model as a fixed effect were compared with models where time was treated as a random effect using a likelihood ratio test.

Dependent Variables: Lying and Standing Times. Generally, calves experiencing more discomfort spend more time standing than lying (Theurer et al., 2012). Within each hour, time spent lying and time spent standing summed to $60 \mathrm{~min}$, so only time spent lying was treated as a dependent variable in the analysis. The median lying time per hour by treatment group preceding and following disbudding was compared using a Kruskal-Wallis rank-sum test followed by a pairwise Wilcoxon rank-sum test with Holm-Bonferroni adjustment for multiple comparisons to allow relative 
comparison of the effect of individual treatments. The median lying time before and after disbudding by treatment group was also compared using a Kruskal-Wallis rank-sum test.

Multivariable linear mixed regression was used to examine the effect of treatment on lying time per calf per hour of the day after disbudding. Treatment group was forced into all models, and independent variable selection was as described previously. Models were constructed where calf was included as a random intercept and observation hour as a random slope to account for repeated observations on the same calf. These were compared with models where calf and observation time were treated as fixed effects with the model selected with the lowest AIC.

All calves were disbudded within $3 \mathrm{~h}$ of $1200 \mathrm{~h}$, so the effect of time of observation could not be differentiated into the effect of time from disbudding compared with the effect of time of day. A quadratic transformation of observation time was used because the association between observation time and lying time was nonlinear, with a peak between 1800 and $0600 \mathrm{~h}$. Within the model, for each calf-hour observation of time spent lying postdisbudding, the corresponding calf-hour of time spent lying in the $24 \mathrm{~h}$ preceding disbudding was included as an independent variable. The proportion of the total variance for repeat measures on the same calf and in the same observation hour was calculated as the intraclass correlation coefficient. All regression models were tested using standard diagnostic techniques for homoscedasticity, normality of residuals, linearity of predictor-outcome association, and the effect of outliers.

\section{RESULTS}

\section{Distribution of Variables Across Treatment Groups}

There was no difference in the distribution of the independent variables across the treatment groups, with a mean age of $31 \mathrm{~d}(95 \% \mathrm{CI}=28-34)$, mean proportion of Friesian genetics of $0.6(95 \%$ CI $=0.55-0.62)$, and mean proportion of male calves of $0.2(95 \% \mathrm{CI}=$ 0.03-0.36). Results from all 80 calves were available for analysis. All buds were removed with the disbudding iron alone, and no calves required amputation dehorning.

\section{Time to Skin Desensitization}

Time to skin desensitization was not normally distributed. For each calf, the median and range in time for skin desensitization averaged over both horn buds are reported in Table 3. For calves that received local infiltration, there was no significant difference for the time to skin desensitization between calves that were disbudded and calves that were not disbudded (INFDB vs. INF-NDB, $P=0.715)$. Similarly, for calves that received a cornual nerve block, there was no significant difference for the time to skin desensitization between calves that were disbudded and calves that were not disbudded (CNB-DB vs. CNB-NDB, $P=0.841$ ). All other pairwise comparisons of treatment groups were significantly different $(P<0.001)$. Supplemental Figure S1 (https://doi.org/10.3168/jds.2018-15033) shows a dot plot of the time to skin desensitization for the treatment groups.

For both methods of administering anesthesia, the time to skin desensitization was not influenced by whether the calves were sham disbudded or disbudded. This allowed us to pool the results for time to skin desensitization by method of administration of anesthesia. The median time for skin desensitization for calves receiving local infiltration (60 s; range: 30-105 s) was significantly less than the median time for calves receiving a cornual block (225 s; range: $120-300$ s; $P<$ $0.001)$.

The logistic multivariable regression model (Supplemental Table S1, https://doi.org/10.3168/jds.2018 -15033) for the time to desensitization predicted that

Table 3. Median (range in parentheses) of time to skin desensitization of the horn buds and body reaction score during disbudding over both horn buds in calves that were control handled or injected with local anesthetic using a local infiltration or a cornual nerve block technique before disbudding

\begin{tabular}{|c|c|c|c|c|c|c|}
\hline Item & \multicolumn{5}{|c|}{ Treatment $^{1}$} & $P$-value \\
\hline $\begin{array}{l}\text { Time to skin desensitization }(\mathrm{s}) \\
\text { Body reaction score }(0-3)\end{array}$ & $\begin{array}{l}30^{2}(30-60) \\
0.0(0.0-0.5)\end{array}$ & $\begin{array}{c}60(30-105) \\
0.0(0.0-0.5)\end{array}$ & $\begin{array}{r}233(120-270) \\
0.3(0.0-1.0)\end{array}$ & $\begin{array}{c}60(30-105) \\
0.5(0.0-2.0)\end{array}$ & $\begin{array}{r}218(135-300) \\
1.0(0.0-3.0)\end{array}$ & $\begin{array}{l}<0.001^{3} \\
<0.001^{3}\end{array}$ \\
\hline
\end{tabular}




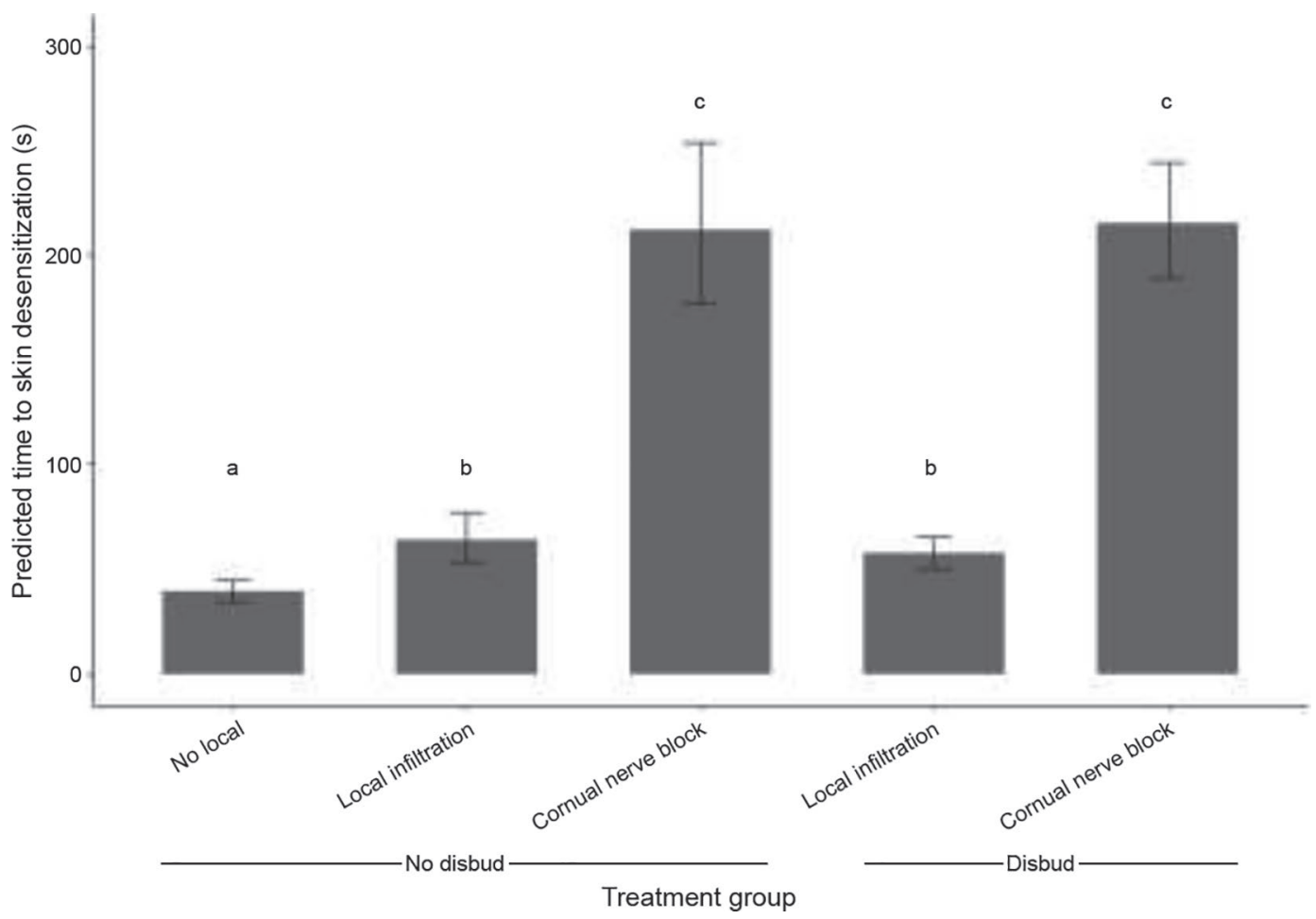

Figure 2. Median and interquartile range of time to skin desensitization predicted by the multivariable logistic regression model from data collected in this study. Columns with different letters $(\mathrm{a}-\mathrm{c})$ are significantly different $(P<0.001)$. Error bars $=\mathrm{IQR}$.

the median time for desensitization under cornual block was 210 s (interquartile range: 180-240) compared with 60 s (interquartile range: $30-60)$ for desensitization under local infiltration $(P<0.001)$. Figure 2 shows the median and interquartile time to skin desensitization as predicted by this model.

The model suggests that for both methods of anesthesia, time for skin desensitization was not influenced by whether the calves were sham disbudded or disbudded. The median time for skin desensitization for calves given local infiltration and disbudded (60 s: interquartile range: $30-60 \mathrm{~s}$ ) was less than that for calves given a cornual block and disbudded (210 s: interquartile range: $180-240 \mathrm{~s} ; P<0.001$ ).

\section{Behavior During Disbudding}

Only 3 calves vocalized and 1 calf defecated during disbudding (all within the group disbudded using the cornual nerve block method), so statistical analysis of these outcomes was not attempted. Median body reaction and range of score for each treatment group are presented in Table 3. Interobserver agreement for the behavior during disbudding was high, with a Spearman rank coefficient of 0.96 for farm A and 0.98 for farm B. For calves that were not disbudded, injecting anesthetic using the infiltration method led to no greater body reaction than injecting using the cornual block method $(P=0.537)$, but there was a trend for an increase in body reaction for calves injected with a cornual block compared with noninjected calves $(P=0.054)$.

Calves that were disbudded under cornual block had a greater body response than calves that were only injected with a cornual block $(P=0.010)$ and a greater body response than calves disbudded under local infiltration $(P=0.029)$. Calves that were disbudded under local infiltration tended to have a greater body response than calves that were only injected with local infiltration $(P=0.051)$. Supplemental Figure S2 (https://doi.org/10.3168/jds.2018-15033) shows a dot plot of the recorded body responses for the treatment groups.

The median body response of calves disbudded under a cornual block (1.0; range: 0.0-3.0) was significantly greater than the body response of calves disbudded under local infiltration $(0.0$; range: $0.0-2.0 ; P=0.005)$. Irrespective of method of administering local anesthetic, disbudding increased the median body response compared with nondisbudded calves (median: 0.0; range: $0.0-1.0 ; P<0.001)$.

There was no difference in the proportion of successful anesthesia (body response score of 1.0 or less) 
achieved between the control groups, so the control groups of nondisbudded calves were pooled. Successful anesthesia was achieved in all control calves $(\mathrm{n}=40)$ and in 18 out of 20 (90\%; 95\% CI $=76.9-100 \%)$ calves disbudded under local infiltration $(P=0.107)$. Successful anesthesia was achieved in 11 out of $20(55 \%$; $95 \% \mathrm{CI}=33.2-76.8 \%$ ) calves disbudded under cornual block. This was significantly less than for control calves $(P<0.001)$ and less than for calves disbudded under local infiltration $(P=0.047)$. Seventeen out of $20(85 \%$; $95 \% \mathrm{CI}=69.4-100 \%)$ calves disbudded under cornual block and 19 out of 20 (95\%; 95\% CI $=85.4-100 \%)$ calves disbudded under local infiltration $(P=0.322)$ had a body response of $<2$.

A multivariable ordinal logistic model for body response score with a probit link had the lowest AIC value (Table 4). Only treatment group was significant in the final model $(P<0.001)$. Because there was no significant difference between the control groups, these were pooled as defined above as nondisbudded calves $(\mathrm{n}=40)$.

The coefficients from the model indicate that calves disbudded under local infiltration or under cornual block are significantly more likely to have a higher body response score than calves in the control group $(P<0.001)$. In a separate analysis (not shown), the referent category was changed to calves disbudded under cornual block. The coefficient for calves disbudded under local infiltration was $-1.03(95 \%$ CI $=-1.72$ to $-0.35 ; P<0.001)$ compared with calves disbudded under cornual block. This indicates that the body response was significantly less for calves disbudded under local infiltration than for calves disbudded under cornual block. The predicted probability of each category of body response score is shown in Supplemental Figure S3 (https://doi.org/10.3168/jds.2018-15033), and the predicted probability that the body response score will be less than or equal to a given value is plotted in Figure 3.

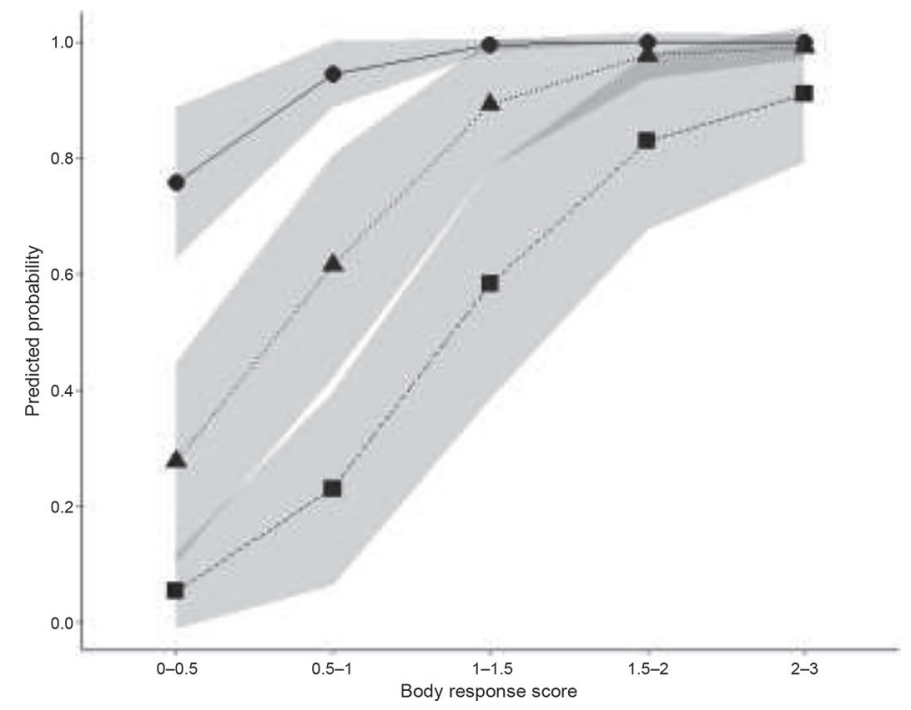

Figure 3. Predicted probability that the body response score will be less than or equal to a given value for the 3 treatment groups from a multivariable ordinal probit model used in this study. Solid circles represent control calves that were not disbudded, solid triangles represent calves that were disbudded under local infiltration, and solid squares represent calves disbudded under cornual block.

Time between administration of local anesthetic and application of disbudding iron was considered as a potential confounder because it was related to treatment group (CNB calves had a longer average time interval than INF calves) and to the body reaction (unadjusted body reaction odds ratio for a 1-unit increase in the time interval $=1.01 ; 95 \% \mathrm{CI}=1.01-1.01)$.

When time to effect was included as a confounding variable in the model, there was a change in the coefficient values of $<20 \%$ and no change in the predicted probability for each body response score $(P>0.89)$. However, the predicted probability of an adverse body reaction score for infiltrated calves increased slightly, whereas the predicted probability for cornual block calves decreased slightly (Supplemental Figure S4,

Table 4. Coefficients from a multivariable ordinal probit model predicting the probability of a body response of 0 to 3

\begin{tabular}{lcccc}
\hline Variable & Coefficient & SE & $95 \%$ CI & $P$-value \\
\hline $\begin{array}{l}\text { Treatment group* } \\
\quad \text { Not disbudded }\end{array}$ & $\begin{array}{c}1 \\
\text { Local infiltration + disbud }\end{array}$ & & & \\
$\quad$ Cornual block + disbud & 1.29 & 0.33 & $0.66-1.93$ & $<0.001$ \\
Intercept & 2.32 & 0.36 & $1.62-3.03$ & $<0.001$ \\
$\quad$ Body response 0-0.5 & 0.70 & 0.21 & $0.28-1.11$ & $<0.001$ \\
Body response 0.5-1 & 1.58 & 0.26 & $1.08-2.09$ & $<0.001$ \\
Body response 1-1.5 & 2.53 & 0.33 & $1.89-3.17$ & $<0.001$ \\
Body response 1.5-2 & 3.27 & 0.39 & $2.51-4.04$ & $<0.001$ \\
Body response 2-3 & 3.66 & 0.45 & $2.78-4.54$ & $<0.001$ \\
\hline
\end{tabular}

${ }^{1}$ Significance of coefficient in the model.

* Overall significance of treatment $P<0.001$. 
https://doi.org/10.3168/jds.2018-15033). Thus, in this model, there was no significant difference between the body reaction scores of calves disbudded under infiltration or cornual block.

The multiple intercepts for body response reflect the fact that the probability of a response of 0.0 to 0.5 is less than the probability of a response of 0.0 to 1.0 and less than the probability of a response of 0.0 to 1.5 , and so on. The assumption that the coefficient values were appropriate across all levels of the ordinal response (parallel regression assumption) means that the increase in body response score associated with disbudding is equivalent across all levels of body score response.

The probability of a score below 1.0 to 1.5 is significantly greater for calves that are not disbudded or disbudded under local infiltration compared with calves disbudded under cornual block $(P<0.05)$. There was no significant difference between treatment groups in the probability of calves having a body reaction score of 1.5 to 2 or greater.

The probabilities were also used to predict the expected mean score for each treatment group; these are shown in Figure 4, together with the recorded mean treatment sores. Mean predicted body response of calves disbudded under a cornual block $(1.2 ; 95 \%$ CI $=0.92-1.48)$ was significantly greater than the body response of calves disbudded under local infiltration $(0.6 ; 95 \% \mathrm{CI}=0.40-0.86 ; P=0.005)$. For both groups of disbudded calves, the median body response was significantly greater than for calves that were handled identically but otherwise not disbudded $(0.15 ; 95 \%$ CI $=0.06-0.24 ; P<0.001)$.

\section{Behavior in the $3 \mathrm{~h}$ After Disbudding}

Total Count of Behaviors. The total number of times that each behavior was recorded in the $3 \mathrm{~h}$ after disbudding for each treatment group is presented in Table 5. $P$-values for a univariable group comparison test within each treatment group are not presented because of repeated observations within calf.

Total Count of Head Shaking. In the analysis of the total number of observed behaviors, only the number of head shakes was significantly related to treatment group $(P=0.023)$, and a negative binomial model fit the data better than a Poisson model $(P<$ 0.001 ; overdispersion parameter $=0.81 ; \mathrm{SE}=0.51$ ) Calf sex and farm of origin were forced into the model as suspected confounders. The results of the model are presented in Table 6 .

The model predicted that the rate of head shaking would be significantly greater in all treatment groups compared with the control group that was not given local or disbudded. Figure 5 shows the predicted and actual counts of head shakes for each treatment group together with the Tukey adjusted $95 \%$ confidence intervals.

\section{Behavior Every 20 Min After Disbudding}

Head Shaking. Only head shaking behavior was significantly different between treatment groups $(P$ $=0.022$ ) when recorded and analyzed every $20 \mathrm{~min}$. There was no difference in the likelihood of a Poisson model compared with a negative binomial $(P=1.0$; overdispersion parameter $=0.002 ; \mathrm{SE}=0.0001)$, but the Poisson model was selected on the basis of a lower AIC value. Calf sex and farm of origin were forced into the model as suspected confounders. The results of the model are presented in Supplemental Table S2 (https:/ /doi.org/10.3168/jds.2018-15033).

The variance within each calf for the number of head shakes recorded at each time was high (0.104) compared with the variance between calves $(0.005)$. Correspondingly, the intraclass correlation coefficient was $0.05(95 \% \mathrm{CI}=0.009-0.109)$, suggesting that clustering was having only a minimal effect on the standard errors of the coefficients. The model with observation time included as a fixed effect fit the data better $(P=0.002)$ than a model with a random slope for observation time.

The model predicted that adjusting for all time observations and when all other covariates are at a value of zero, disbudded calves shake their heads more than calves that are not disbudded. Supplemental Figure S5

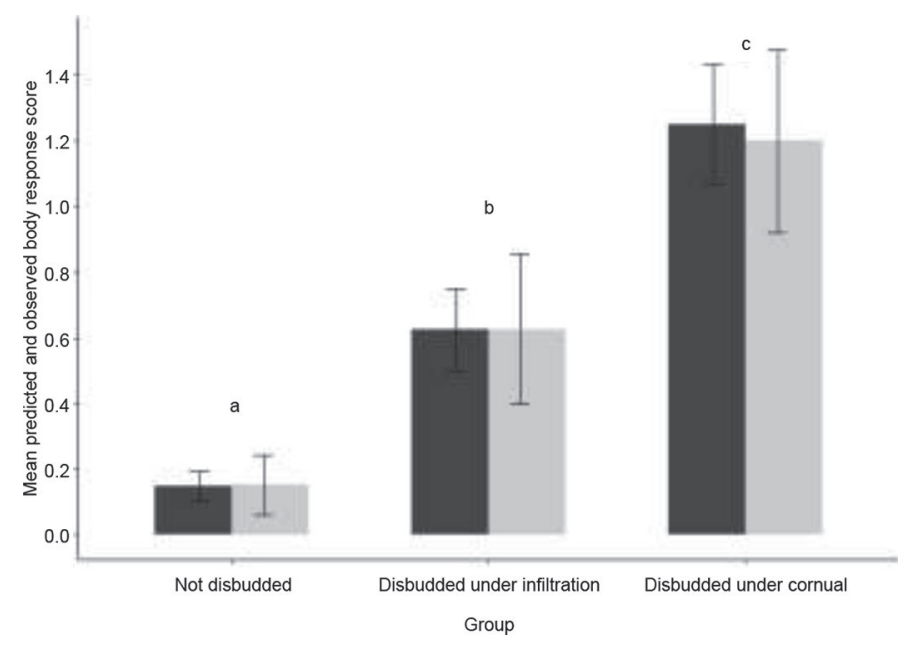

Figure 4. Predicted (gray bars) and actual (black bars) mean body response score for the 3 treatment groups from a multivariable ordinal probit model used in this study. Error bars represent Tukey-adjusted 95\% CI for the mean. Letters $(\mathrm{a}-\mathrm{c})$ indicate difference between treatments. 
Table 5. Total counts (no.) and frequency (\%;95\% CI in parentheses) of each behavior in the $3 \mathrm{~h}$ after disbudding

\begin{tabular}{|c|c|c|c|c|c|}
\hline Item & \multicolumn{5}{|c|}{ Treatment $^{1}$} \\
\hline \multicolumn{6}{|l|}{ Head shake } \\
\hline Count & 6 & 8 & 8 & 26 & 24 \\
\hline Frequency & $0.3(0.11-0.65)$ & $0.8(0.35-1.58)$ & $0.8(0.35-1.58)$ & $1.3(0.85-1.90)$ & $1.2(0.77-1.79)$ \\
\hline \multicolumn{6}{|l|}{ Head scratch } \\
\hline \multicolumn{6}{|l|}{ Head rub } \\
\hline Count & 4 & 3 & 1 & 1 & 0 \\
\hline Frequency & $0.2(0.05-0.51)$ & $0.3(0.06-0.88)$ & $0.1(0.00-0.56)$ & $0.05(0.00-0.28)$ & \\
\hline \multicolumn{6}{|l|}{ Self-groom } \\
\hline Count & 30 & 12 & 9 & 17 & 23 \\
\hline Frequency & $1.5(1.01-2.14)$ & $1.2(0.62-2.10)$ & $0.9(0.41-1.71)$ & $0.85(0.50-1.36)$ & $1.2(0.73-1.73)$ \\
\hline \multicolumn{6}{|l|}{ Running } \\
\hline
\end{tabular}

${ }^{1} \mathrm{CON}=$ no local, no disbud (control); INF-NDB = local infiltration, no disbud; CNB-NDB = cornual block, no disbud; INF-DB = local infiltration, disbud; CNB-DB = cornual nerve block, disbud.

(https://doi.org/10.3168/jds.2018-15033) shows the predicted and actual counts for each treatment group at each time of observation together with the Tukey adjusted $95 \%$ confidence intervals.

Allowing for the effects of sex, farm, treatment group, and observation time, calves that are disbudded shake their heads significantly more often at the first observation $20 \mathrm{~min}$ after disbudding than calves that are not disbudded $(P<0.001)$. There was no significant interaction between observation time and treatment $(P=0.229)$, in agreement with the coefficients in Supplemental Table S2 (https://doi.org/10 $.3168 /$ jds.2018-15033) and the predicted probabilities in Supplemental Figure S5 (https://doi.org/10.3168/ jds.2018-15033).

\section{Lying Time Before and After Disbudding}

The median time spent lying across all groups after disbudding (45 min; range $=0-60$ ) was more than the median time lying before disbudding (41 min; range $=0-60 ; P<0.001)$. However, there was no difference in the median time per hour spent lying by treatment group before $(P=0.720)$ or after $(P=0.927)$ disbudding. The median time per hour spent lying by treatment group before and after disbudding is shown in Supplemental Table S3 (https://doi.org/10.3168/jds .2018-15033).

In the multivariable mixed model, only the time of day and the time spent lying before disbudding were significant. Treatment group was not significant $(P=$

Table 6. Negative binomial model predicting the change in the log of the number of counts of head shaking in the $3 \mathrm{~h}$ after disbudding (95\% CI in parentheses)

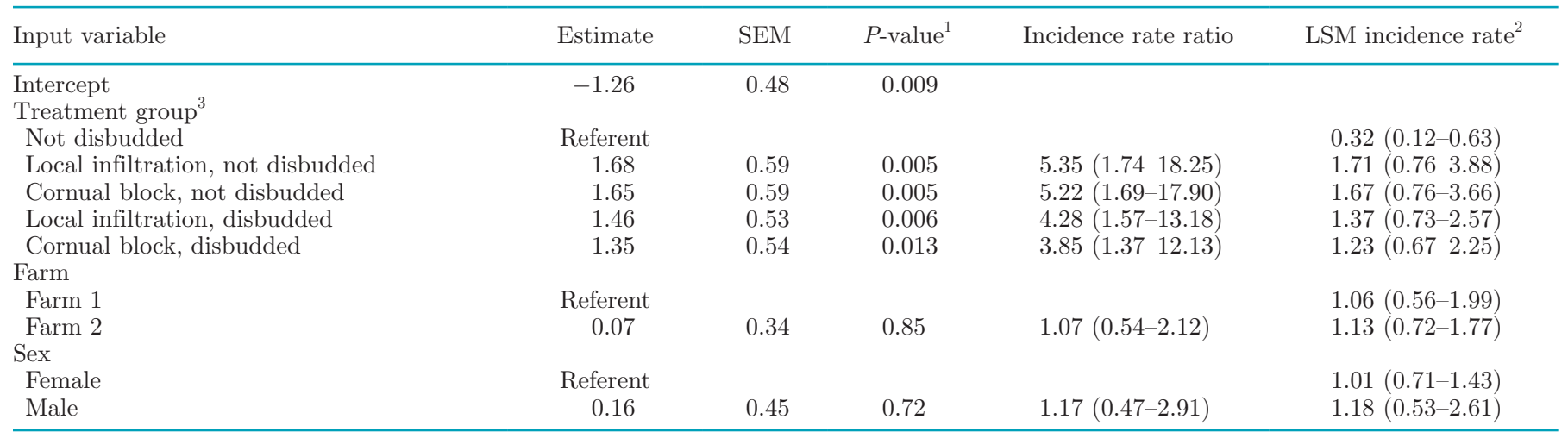

${ }^{1}$ Significance of coefficient in the model.

${ }^{2}$ Least squares means prediction from the regression model.

${ }^{3}$ Overall significance of treatment in the model $P=0.023$. 
0.678 ), and there were no significant interactions in the model. The results for this model are presented in Supplemental Table S4 (https://doi.org/10.3168/jds .2018-15033). Model prediction of the lying time per hour for the $24 \mathrm{~h}$ after disbudding is plotted against the observed data in Supplemental Figure S6 (https://doi .org/10.3168/jds.2018-15033).

\section{DISCUSSION}

The results from our study suggest that the local infiltration block method may be a viable alternative to the cornual nerve block method for the provision of anesthesia for calf disbudding. Compared with a cornual block, we found that onset of anesthesia was faster and less varied, pain response during disbudding was no greater, pain responses in the $3 \mathrm{~h}$ following disbudding were equivalent, and lying behavior associated with pain was equivalent for the $24 \mathrm{~h}$ after disbudding. Producers' ability to implement a change is a key element to adopting new techniques (Jansen et al., 2009; Sorge et al., 2010). We believe the ease of administration and the faster onset produced by this alternative method of anesthetic administration will be more attractive to on-farm users and will promote greater use of local anesthetic for disbudding.

We acknowledge, though, that one of the potential limitations of this study was the needle prick test used to confirm effective anesthesia. Calves undergoing CNB had a greater time to effect than INF calves and therefore received more needle pricks and were restrained for longer $(P<0.001)$. Altough this was done to ensure that all animals were adequately anesthetized before disbudding, it meant that some animals received more needle pricks than others. If repeated needle pricks acted as a source of anxiety for the calves, this could potentially have confounded the relationship between the method of local anesthetic administration and the outcomes of the study. However, the needle prick test was designed to cause an aversive response rather than a painful one in the present study, and we do not believe this test would have been sufficient to lead to aversive anticipation in the calves. Moreover, there was no difference in the time to effect between CNB calves that were disbudded and CNB calves that were not disbudded $(P=1.0)$, meaning that all CNB calves received an

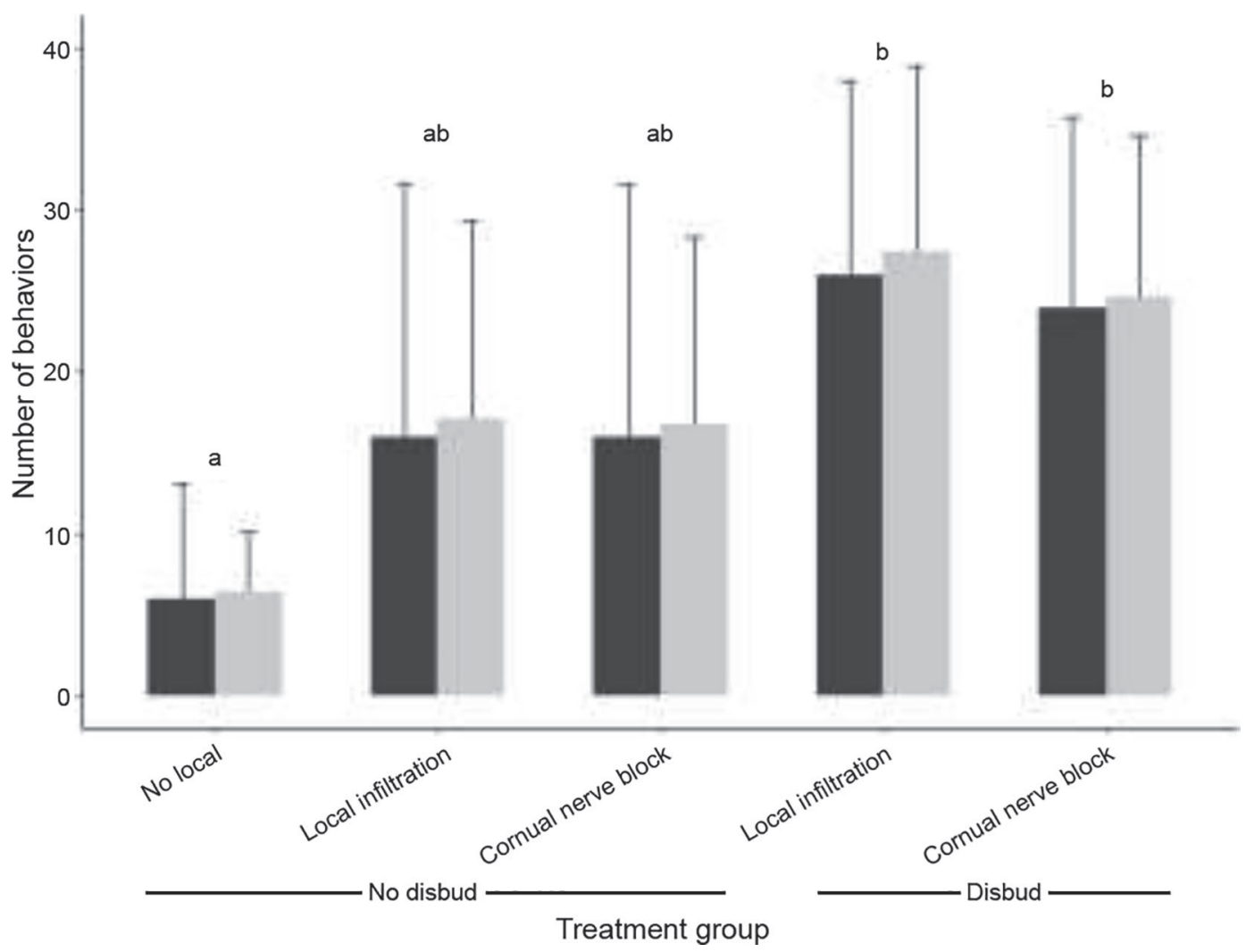

Figure 5. Predicted (gray bars) and actual (black bars) count of head shakes for each treatment group together with the Tukey adjusted 95\% confidence intervals. Different letters $(\mathrm{a}, \mathrm{b})$ indicate a difference $(P<0.05)$ for a pairwise comparison between the predicted values for each group. 
equal number of needle pricks and were restrained for the same time. Despite this, CNB calves that were disbudded had a greater median body response than CNB calves that were not disbudded $(P=0.010)$, suggesting that the difference in body response was not due to the increase in restraint time or needle pricks. We believe this makes it unlikely that the difference in body response between calves disbudded under $\mathrm{CNB}$ and calves disbudded under INF $(P=0.029)$ was attributable to differences in the time to effect. Furthermore, studies evaluating anxiety and central sensitization in animals and humans generally repeat the stimulus at much shorter intervals than $30 \mathrm{~s}$ (Herrero et al., 2000; Coste et al., 2008), which may give the animals and the nervous system more time to recover.

Although in the field it is unlikely that calves will receive multiple needle pricks before disbudding, it is still the case that calves disbudded under cornual block will require a longer wait time between administration of local anesthetic and disbudding. Stewart et al. (2008) recommended a wait period of $10 \mathrm{~min}$ for the local anesthetic to be effective, which will involve either restraint in the handling facility for this time or double handling of each calf. When we attempted to adjust for time to effect analytically (Jager et al., 2008), inclusion of time to effect changed the model coefficients by $<20 \%$. Although the probability of an adverse response for cornual block calves decreased when time was included, there was no statistical difference in the predicted probability of an adverse response $(P=0.763)$.

In this situation, our null hypothesis was that there is no difference in the coefficients regardless of whether time to effect is included. Simulation studies allowing for the odds ratio, sample size, and correlation between time and group indicated that to keep the probability of a type 1 error $<5 \%$ and maintain power above $80 \%$, for variables to be treated as confounders they would need to induce a change in coefficient values greater than $30 \%$ (Lee, 2014).

Consequently, we treated time to effect as an intermediary variable (Chaiton et al., 2015) lying on the casual pathway between exposure and outcome (Greenland, 2008). In these circumstances, including intermediary variables leads to overadjustment bias and an inappropriate downward adjustment of the effect of treatment (Hernández-Diaz et al., 2008; Schisterman et al., 2009; Ananth and Schisterman, 2017).

The cornual nerve block has been recommended over a ring block (Winder et al., 2017) because of a greater duration of anesthesia after the procedure (Fierheller et al., 2012). In addition, the deposition of local anesthetic proximal to the site of disbudding may lead to a poorer effect because local anesthetic (e.g., lidocaine) is less likely to dissociate to its active form in an acidic environment resulting from tissue damage (Anderson and Edmondson, 2013). Given that anesthesia from lidocaine persists for approximately $2 \mathrm{~h}$ (Sutherland et al., 2002) irrespective of how it is administered, we would recommend the addition of a nonsteroidal antiinflammatory drug at disbudding to both the local infiltration and cornual block in accordance with Stafford and Mellor (2011) and Stock et al. (2013).

We did not observe any difference in the $3 \mathrm{~h}$ or the $24 \mathrm{~h}$ after disbudding in calf behavior. Our presample power calculation suggested that we would have had power in excess of $80 \%$ for observation of head shakes and scratches as well as self-grooming and running (Table 5). In addition, our number of study animals was comparable with those used in other papers $(8$ calves per group in McMeekan et al., 1999; 6 calves per group in Theurer et al., 2012; and 10 calves per group in Faulkner and Weary, 2000). We cannot rule out a lack of power in our present study, but we suggest that any difference in the postoperative behavior between calves disbudded with a cornual block versus a local infiltration block is small.

Although we had high interobserver agreement for the measure of calf behavior during disbudding, a lack of available trained staff meant that we were not able to measure interobserver agreement for the $3 \mathrm{~h}$ of observation after disbudding. However, as all observes were blind to treatment status, any bias in observation would have been evenly distributed between the treatment groups.

The efficacy of the cornual block in our study was less than the 75 to $91 \%$ reported by Winder et al. (2018) and the $87.5 \%$ reported by Fierheller et al. (2012). In our study, the person administering the blocks was not blind to the treatment status during the analysis period, and it is possible that lack of operator skill or familiarity with the technique could have been a factor. Qualification status and length of time qualified is no guarantee that a cornual block will be administered correctly. However, the question becomes, if lack of skill was a factor, how well would relatively inexperienced farm staff manage this task?

In their study looking at the effect of different training methods, Winder et al. (2018) demonstrated that 75 to $91 \%$ of farm staff with no previous experience could achieve an effective cornual block in a single calf when the evaluation period was the first $5 \mathrm{~s}$ of disbudding and the time between injecting the cornual block and disbudding was standardized to $10 \mathrm{~min}$. We observed some calves responding adversely to disbudding as soon as the disbudding iron was applied, whereas others would show a negative response only when the 
horn bud was scooped out at the end of the procedure. Potentially, this latter group would not have been recorded as a negative reaction when only the first $5 \mathrm{~s}$ of disbudding was scored; therefore, our recording technique may have been more sensitive. We also reported greater range in the body reaction under cornual block compared with local infiltration block. This suggests that successful evaluation should be based on observation of multiple measures of pain in response to disbudding.

We also reported a greater range in the time to skin desensitization under the cornual block compared with the local infiltration block. Time of onset for lidocaine is 2 to $5 \mathrm{~min}$ (Coetzee, 2013). Rapid onset of action with a cornual nerve block is dependent on proximity of placement of the lidocaine to the branches of the cornual nerve, with delay or failure associated with deposition in the muscle, deep to the nerve fibers (Skarda, 1996; Anderson and Edmondson, 2013). We suggest that the time delay between administration of the block and disbudding is critical for its efficacy. This needs to be factored into training along with an effective means of assessing block efficacy, such as a blink response during administration of the block and drooping of the eyelid thereafter (Skarda, 1996). In the current study, all calves were assessed for skin sensitivity to needle prick before disbudding, but some disbudded calves still had a body reaction score higher than control calves. As a test for anesthesia for hot iron disbudding, absence of response to needle prick may overestimate the degree of block.

Whether time to effect is treated as a confounding or intermediary variable, these results suggest that the local infiltration block is as effective as the cornual block method for alleviating the immediate pain associated with disbudding. Further work on farmer training in the use of the local infiltration and cornual block is warranted to establish a method of delivering effective pain relief on farm. Additional methods of recording nociceptive perception such as infrared thermography (Stewart et al., 2009), heart rate variability (Stewart et al., 2008), and response to mechanical or electrical stimulation (Heinrich et al., 2010) should be applied to calves disbudded with the local infiltration block to ensure that they are not worse off postoperatively than calves disbudded under a cornual block.

Reliable local anesthesia for calf disbudding is increasingly recognized as an industry requirement. The local infiltration block method appears to represent a viable alternative to the cornual block method and may have some practical advantages for use by nonveterinarians. Further work is required to evaluate the efficacy of nerve blocks when applied by farmers.

\section{ACKNOWLEDGMENTS}

This work was funded by AgMardt NZ (Fielding, New Zealand; reference AIGItIn-000272). Data loggers were provided by AgResearch (Hamilton, New Zealand), and training in observation of calf behavior was provided by Suzanne Dowling (AgResearch). We acknowledge all the farmers and their staff for their help and enthusiasm with this trial and all Vetlife Temuka (Temuka, New Zealand) staff. We also acknowledge the support of Vetlife management in supporting practicebased research. Comments from Richard Laven (School of Veterinary Science, Massey, New Zealand) helped improve the manuscript.

\section{REFERENCES}

Adams, A., J. Lombard, C. Shivley, N. Urie, I. Roman-Muniz, C. Fossler, and C. Kopral. 2015. Management practices that may impact dairy heifer welfare on U.S. dairy operations. Page 105 in Proceedings of the Joint Annual Meeting of the American Dairy Science Association. ADSA, Champaign, IL.

Ananth, C. V., and E. F. Schisterman. 2017. Confounding, causality, and confusion: The role of intermediate variables in interpreting observational studies in obstetrics. Am. J. Obstet. Gynecol. 217:167-175.

Anderson, D. E., and M. Edmondson. 2013. Prevention and management of surgical pain in cattle. Vet. Clin. North Am. Food Anim. Pract. 29:157-184.

Bates, A., P. Eder, and R. Laven. 2015. Effect of analgesia and antiinflammatory treatment on weight gain and milk intake of dairy calves after disbudding. N. Z. Vet. J. 63:153-157. https://doi.org/ 10.1080/00480169.2014.982739.

Bates, A. J., R. Laven, F. Chapple, and D. Weeks. 2016. The effect of different combinations of local anaesthesia, sedative and nonsteroidal anti-inflammatory drugs on daily growth rates of dairy calves after disbudding. N. Z. Vet. J. 64:282-287. https://doi.org/ 10.1080/00480169.2016.1196626.

Bonk, S., O. Burfeind, V. Suthar, and W. Heuwieser. 2013. Technical note: Evaluation of data loggers for measuring lying behavior in dairy calves. J. Dairy Sci. 96:3265-3271.

Chaiton, M., J. E. Cohen, J. Rehm, M. Abdulle, and J. O'Loughlin. 2015. Confounders or intermediate variables? Testing mechanisms for the relationship between depression and smoking in a longitudinal cohort study. Addict. Behav. 42:154-161.

Coetzee, J. F. 2013. A review of analgesic compounds used in food animals in the United States. Vet. Clin. North Am. Food Anim. Pract. 29:11-28. https://doi.org/10.1016/j.cvfa.2012.11.008.

Coste, J., D. Voisin, P. Luccarini, and R. Dallel. 2008. A Role for windup in trigeminal sensory processing: Intensity coding of nociceptive stimuli in the rat. Cephalalgia 28:631-639. https://doi.org/10 $.1111 / \mathrm{j} .1468-2982.2008 .01568 . x$.

Croney, C. C., and R. Anthony. 2010. Engaging science in a climate of values: Tools for animal scientists tasked with addressing ethical problems. J. Anim. Sci. 88:E75-E81.

Faulkner, P. M., and D. Weary. 2000. Reducing pain after dehorning in dairy calves. J. Dairy Sci. 83:2037-2041.

Fierheller, E. E., N. Caulkett, D. Florence, D. Haley, and L. Doepel. 2012. Onset, duration and efficacy of four methods of local anesthesia of the horn bud in calves. Vet. Anaesth. Analg. 39:431-435.

Gottardo, F., M. Brscic, B. Contiero, N. Irrgang, U. Knierim, O. Pentelescu, J. Windig, L. Mirabito, F. Kling Eveillard, A. Dockes, I. Veissier, A. Velarde, C. Fuentes, A. Dalmau, and C. Winckler. 2015. Dehorning of cattle in the EU Member States: A quantita- 
tive survey of the current practices. Livest. Sci. 179:4-11. https:// doi.org/10.1016/J.LIVSCI.2015.05.011.

Graf, B., and M. Senn. 1999. Behavioural and physiological responses of calves to dehorning by heat cauterization with or without local anaesthesia. Appl. Anim. Behav. Sci. 62:153-171.

Green, T. C., and D. J. Mellor. 2011. Extending ideas about animal welfare assessment to include "quality of life" and related concepts. N. Z. Vet. J. 59:263-271.

Greenland, S. 2008. Invited commentary: Variable selection versus shrinkage in the control of multiple confounders (American Journal of Epidemiology (2008) 167 (523-529). Am. J. Epidemiol. 167:1142. https://doi.org/10.1093/aje/kwn088.

Grøndahl-Nielsen, C., H. Simonsen, J. Lund, and M. Hesselholt. 1999 Behavioural, endocrine, and cardiac responses in young calves undergoing dehorning without and with use of sedation and analgesia. Vet. J. 158:14-20.

Harrell, F. 2001. Regression Modeling Strategies. 1st ed. Springer, New York, NY.

Heinrich, A., T. Duffield, K. Lissemore, and S. Millman. 2010. The effect of meloxicam on behavior and pain sensitivity of dairy calves following cautery dehorning with a local anesthetic. J. Dairy Sci. 93:2450-2457. https://doi.org/10.3168/jds.2009-2813.

Heinze, G., and M. Schemper. 2002. A solution to the problem of separation in logistic regression. Stat. Med. 21:2409-2419.

Hernández-Diaz, S., A. Wilcox, E. Schisterman, and M. Hernan. 2008. From causal diagrams to birth weight-specific curves of infant mortality. Eur. J. Epidemiol. 23:163-166.

Herrero, J. F., J. M. A. Laird, and J. A. Lopez-Garcia. 2000. Windup of spinal cord neurones and pain sensation: Much ado about something? Prog. Neurobiol. 61:169-203.

Jager, K. J., C. Zoccali, A. MacLeod, and F. W. Dekker. 2008. Confounding: What it is and how to deal with it. Kidney Int. 73:256260. https://doi.org/10.1038/sj.ki.5002650.

Jansen, J., B. H. P. van den Borne, R. J. Renes, G. van Schaik, T. J. G. M. Lam, and C. Leeuwis. 2009. Explaining mastitis incidence in Dutch dairy farming: The influence of farmers' attitudes and behaviour. Prev. Vet. Med. 92:210-223.

Lee, P. H. 2014. Is a cutoff of $10 \%$ appropriate for the change-in-estimate criterion of confounder identification? J. Epidemiol. 24:161167. https://doi.org/10.2188/jea.JE20130062.

Lehr, R. 1992. Sixteen S-squared over D-squared: A relation for crude sample size estimates. Stat. Med. 11:1099-1102.

Lundmark, F., C. Berg, O. Schmid, D. Behdadi, and H. Röcklinsberg. 2014. Intentions and values in animal welfare legislation and standards. J. Agric. Environ. Ethics 27:991-1017.

McMeekan, C., K. Stafford, R. Bruce, R. Ward, D. Mellor, and N. Gregory. 1999. Effects of a local anaesthetic and a non-steroidal anti-inflammatory analgesic on the behavioural responses of calves to dehorning. N. Z. Vet. J. 47:92-96. https://doi.org/10.1080/ 00480169.1999.36120.

Mellor, D. J., and K. Stafford. 2004. Animal welfare implications of neonatal mortality and morbidity in farm animals. Vet. J. 168:118-133.

Petrie, N. J., D. Mellor, K. Stafford, R. Bruce, and R. Ward. 1996. Cortisol responses of calves to two methods of disbudding used with or without local anaesthetic. N. Z. Vet. J. 44:9-14.

Robbins, J., D. Weary, C. Schuppli, and M. von Keyserlingk. 2015. Stakeholder views on treating pain due to dehorning dairy calves. Anim. Welf. 24:399-406.
Schisterman, E. F., S. Cole, and R. Platt. 2009. Overadjustment bias and unnecessary adjustment in epidemiologic studies. Epidemiology 20:488-495.

Skarda, R. T. 1996. Local and regional anesthesia in ruminants and swine. Vet. Clin. North Am. Food Anim. Pract. 12:579-626.

Sorge, U., D. Kelton, K. Lissemore, A. Godkin, S. Hendrick, and S. Wells. 2010. Attitudes of Canadian dairy farmers toward a voluntary Johne's disease control program. J. Dairy Sci. 93:1491-1499. https://doi.org/10.3168/jds.2009-2447.

Stafford, K. J., and D. Mellor. 2005. Review: Dehorning and disbudding distress and its alleviation in calves. Vet. J. 169:337-349.

Stafford, K., and D. Mellor. 2011. Addressing the pain associated with disbudding and dehorning in cattle. Appl. Anim. Behav. Sci. 135:226-231.

Stewart, M., K. Stafford, S. Dowling, A. Schaefer, and J. Webster. 2008. Eye temperature and heart rate variability of calves disbudded with or without local anaesthetic. Physiol. Behav. 93:789-797.

Stewart, M., J. Stookey, K. Stafford, C. Tucker, A. Rogers, S. Dowling, G. Verkerk, A. Schaefer, and J. Webster. 2009. Effects of local anesthetic and a nonsteroidal antiinflammatory drug on pain responses of dairy calves to hot-iron dehorning. J. Dairy Sci 92:1512-1519.

Stilwell, G., M. Lima, and D. Broom. 2007. Comparing the effects of three different disbudding methods on behavior and plasma cortisol of calves. Rev. Port. Ciências Veterinárias 102:281-288.

Stock, M. L., S. Baldridge, D. Griffin, and J. Coetzee. 2013. Bovine dehorning: Assessing pain and providing analgesic management. Vet. Clin. North Am. Food Anim. Pract. 29:103-133.

Sutherland, M. A., D. Mellor, K. Stafford, N. Gregory, R. Bruce, and R. Ward. 2002. Cortisol responses to dehorning of calves given a 5-h local anaesthetic regimen plus phenylbutazone, ketoprofen, or adrenocorticotropic hormone prior to dehorning. Res. Vet. Sci. $73: 115-123$

Sutherland, M. A., G. M. Worth, C. Cameron, C. M. Ross, and D. Rapp. 2017. Health, physiology, and behavior of dairy calves reared on 4 different substrates. J. Dairy Sci. 100:2148-2156. https://doi .org/10.3168/jds.2016-12074.

Theurer, M. E., B. J. White, J. Coetzee, M. Theurer, R. Mosher, and C. Cull. 2012. Assessment of behavioral changes associated with oral meloxicam administration at time of dehorning in calves using a remote triangulation device and accelerometers. BMC Vet. Res. 8:48-56.

UBC AWP. 2015. SOP - HOBO data loggers. Pages 14-30 in UBC Animal Welfare Program, Version 1.

van Belle, G. 2008. Sample Size. 2nd ed. Wiley, Hoboken, NJ.

Ventura, B., M. Keyserlingk, and D. Weary. 2015. Animal welfare concerns and values of stakeholders within the dairy industry. J. Agric. Environ. Ethics 28:109-126.

Winder, C. B., S. LeBlanc, D. Haley, K. Lissemore, T. Duffield, and M. Godkin. 2017. Clinical trial of local anesthetic protocols for acute pain associated with caustic paste disbudding in dairy calves. J. Dairy Sci. 100:6429-6441.

Winder, C. B., S. LeBlanc, D. Haley, K. Lissemore, M. Godkin, and T. Duffield. 2018. Comparison of online, hands-on, and a combined approach for teaching cautery disbudding technique to dairy producers. J. Dairy Sci. 101:840-849. 